\title{
PCR Amplification on a Microarray of Gel- Immobilized Oligonucleotides: Detection of Bacterial Toxin- and Drug-Resistant Genes and Their Mutations
}

BioTechniques 29:844-857(October 2000)

\author{
Boris N. Strizhkov ${ }^{1}$, Alexei L. \\ Drobyshev', Vladimir M. \\ Mikhailovich $^{1}$ and \\ Andrei D. Mirzabekov ${ }^{1,2}$ \\ 'Engelhardt Institute of Molecu- \\ lar Biology, Russian Academy \\ of Sciences, Moscow, Russia \\ and ${ }^{2}$ Argonne National Labora- \\ tory, Argonne, IL, USA
}

\section{ABSTRACT}

PCR amplification on a microarray of gel-immobilized primers (microchip) has been developed. One of a pair of PCR primers was immobilized inside a separate microchip polyacrylamide porous gel pad of $0.1 \times 0.1 \times 0.02($ or 0.04$) \mu \mathrm{m}$ in size and 0.2 (or 0.4) $n L$ in volume. The amplification was carried out simultaneously both in solution covering the microchip array and inside gel pads. Each gel pad contained the immobilized forward primers, while the fluorescently labeled reverse primers, as well as all components of the amplification reaction, diffused into the gel pads from the solution. To increase the amplification efficiency, the forward primers were also added into the solution. The kinetics of amplification was measured in real time in parallel for all gel pads with a fluorescent microscope equipped with a charge-coupled device (CCD) camera. The accuracy of the amplification was assessed by using the melting curves obtained for the duplexes formed by the labeled amplification product and the gel-immobilized primers during the amplification process; alternatively, the duplexes were produced by hybridization of the extended immobilized primers with labeled oligonucleotide probes. The on-chip amplification was applied to detect the anthrax toxin genes and the plasmid-borne $\beta$-lactamase gene responsible for bacterial ampicillin resistance. The allele-specific type of PCR amplification was used to identify the Shiga toxin gene and discriminate it from the Shiga-like one. The genomic mutations responsible for rifampicin resistance of the Mycobacterium tuberculosis strains were detected by the same type of PCR amplification of the rpoBgene fragment isolated from sputum of tuberculosis patients. The on-chip PCR amplification has been shown to be a rapid, inexpensive and powerful tool to test genes responsible for bacterial toxin production and drug resistance, as well as to reveal point nucleotide mutations.

\section{INTRODUCTION}

The need for fast and inexpensive DNA-based medical, environmental and bioterrorist tests for microorganisms has become increasingly apparent. One of the most efficient assays is PCR amplification of the DNA target regions followed by product analysis. This method continues to be refined. For example, both miniaturizing PCR and manufacturing the PCR microchips save in cost of reagents and time of analysis $(3,16$, 25,27). The combined DNA amplification and analysis, as in the TaqMan ${ }^{\circledR}$ (14) and Molecular Beacons approaches (26), would further simplify and accelerate the method. Multiplex amplification dealing with a mixture of many primers is still restricted to a few probes (for a review see References 8 and 24). At the same time, the parallel amplification reactions for a large number of probes are intensively robotized (for a review see Reference 15).

The microarrays of immobilized oligonucleotides called oligonucleotide microchips have been used to detect various bacteria and analyze their genes by hybridization with PCR-amplified DNA [including 16S ribosomal RNA gene (10)] or by hybridization directly with ribosomal RNA (11).

In this paper, we address PCR im provement through the development of PCR amplification on a microarray of gel-immobilized compounds on a chip (MAGIChip $^{\mathrm{TM}}$ ). The MAGIChip, which is produced at both ANL and EIMB, is an array of $0.1 \times 0.1 \times 0.02$ (or 0.04 ) $-\mu \mathrm{m}$ polyacrylamide-based gel pads containing different immobilized oligonucleotides. As many as 4096 of all possible hexadeoxynucleotides were placed on such a chip of $18 \times 21 \mathrm{~mm}$ in size to analyze the sequence specificity of a DNA-binding drug (5) and identify point mutations (21). The microchip gel pads can serve as microtest tubes of $0.2-0.4 \mathrm{~nL}$ in volume and have been used in a number of chemical and enzymatic reactions, such as immobilization of oligonucleotides and DNA through covalent bonds (22), DNA ligation (7) and DNA polymerase extension (6) of immobilized oligonucleotides. Here, we describe a new procedure for bacterial analysis that combines PCR amplification on a MAGIChip, multiplex allelespecific amplification and detection of amplified fluorescently labeled products with a fluorescent microscope. PCR analysis was also carried out in real time to monitor the kinetics of the reaction.

The procedure was applied for detection and mutation analysis of genes of a number of microorganisms of practical importance: the anthrax toxin and Shiga toxin genes, the plasmid-born ampicillin resistance gene and rifam- 
picin-resistant genomic mutations of Mycobacterium tuberculosis. Bacillus anthracis is potentially the most dangerous bioterrorist agent (13). The Shiga and Shiga-like toxin genes of the toxin-producing strains of Shigella dysenteriae and Escherichia coli, respectively, when inhabiting human intestines, are responsible for hemorrhagic colitis and other deadly diseases. The spread of $M$. tuberculosis, including its drug-resistant strains, is growing and is now the most common cause of death from infectious diseases in the world. Until recently, rifampicin was one of the most efficient drugs used to treat tuberculosis patients. Rifampicin resistance, in $96 \%$ of $M$. tuberculosis strains, is caused by mutations occurring within a short region of the $r p o B$ gene $(18,19)$. Detection of a gene encoding $\beta$-lactamase, which inactivates ampicillin, is a good model for screening bacterial plasmids bearing genes of drug resistance.

\section{MATERIALS AND METHODS}

\section{Oligonucleotides, PCR Products and DNA}

The primers for PCR amplification were synthesized with a model 394 DNA/RNA synthesizer (Applied Biosystems, Foster City, CA, USA) using standard phosphoramidite chemistry. Both the primers that were immobilized and those that were fluorescently labeled contained an amino link with a $\mathrm{C}_{6}$ spacer (Glen Research, Sterling, VA, USA) at the $5^{\prime}$ terminal position. Texas $\operatorname{Red}^{\circledR}$ was linked to the $5^{\prime}$ terminal amino groups of the oligonucleotides according to the manufacturer's protocol.

Two B. anthracis PCR products of the regions of the lef gene, encoding the lethal factor, and the pag4 gene, encoding the protective antigen, were obtained from the U.S. Army Medical Research Institute of Infectious Diseases (USAMRIID). The 103-bp lef fragment (nucleotide positions $1153-1255$, GenBank ${ }^{\circledR}$ accession no. m30210) and the 248-bp pag4 fragment (nucleotide positions 1931-2178, GenBank accession no. m22589) were generated by PCR am plification of the $B$. anthracis products.

Plasmid pUC18 (Sigma, St. Louis,
MO, USA) containing an ampicillin resistance gene (bla) was used for PCR amplification of the 2656-2748-bp fragment (GenBank accession no. AF074376). Plasmid pSHT23 (17) containing the Shiga toxin gene $(s h t)$ was used for PCR amplification of the 1619-1757-bp fragment (nucleotide positions 127-265, GenBank accession no. AJ132761).

DNA from different strains of $M$. $t u$ berculosis was isolated from clinical samples of sputum (12) provided by the Moscow Scientific and Clinical AntiTuberculosis Center. A freshly prepared $0.5 \%$ solution of $\mathrm{N}$-acetyl-L-cysteine (NALC) in $2 \% \mathrm{NaOH}$ was mixed with an equal volume of sputum and incubated for $40 \mathrm{~min}$ at room temperature. Five volumes of $0.1 \mathrm{M}$ sodium phosphate buffer, $\mathrm{pH}$ 6.8, were added to the mixture, followed by centrifugation for 30 min at $3000 \times g$ in an Eppendorf ${ }^{\circledR}$ centrifuge. The cell pellet was suspended twice in $1.5 \mathrm{~mL} 10 \mathrm{mM}$ Tris- $\mathrm{HCl}, \mathrm{pH}$ 8.0 , containing $1 \mathrm{mM}$ EDTA and centrifuged. The cells in the pellet were lysed in $50 \mu \mathrm{L} 1 \%$ Triton ${ }^{\circledR} \mathrm{X}-100,1$ mM EDTA, $10 \mathrm{mM}$ Tris-HCl, $\mathrm{pH}$ 8.0, at $95^{\circ} \mathrm{C}$ for $15 \mathrm{~min}$, followed by centrifugation at $10000 \times g$ in an Eppendorf centrifuge. DNA from the supernatant was used directly for PCR amplification of the 2339-2461 fragment of RNA polymerase $\beta$-subunit gene (rpoB) (GenBank accession no. L27989).

\section{Oligonucleotide Microchips}

A microchip for PCR amplification contained about 1 pmol oligonucleotide primers immobilized through their $5^{\prime}$ terminal amino groups inside $100 \times$ $100 \times 20-\mu \mathrm{m}$ polyacrylamide gel pads or $100 \times 100 \times 40-\mu \mathrm{m}$ porous mixed polyacrylamide gel pads spaced by a $200-\mu \mathrm{m}$ hydrophobic glass surface (29). Gels of two different porosities containing active aldehyde groups were used for the microchip manufacturing.

Standard acrylamide gels were prepared as described by Yershov et al. (29). For the porous gels (2), the polymerization solution contained $1.5 \%$ acrylamide, $1.5 \%$ N-acryloyltris-(hydroxymethyl)aminomethane (Bio-Rad Laboratories, Hercules, CA, USA), $0.7 \%$ N,N-(1,2-dihydroxyethylene) bisacrylamide (Sigma), $0.2 \%$ N,N-diallyl- tartardiamide (Bio-Rad Laboratories), $0.1 \% \mathrm{~N}, \mathrm{~N}$-methylene bisacrylamide (Sigma), 0.1 M Na-phosphate, pH 7.0, $1.2 \%$ TEMED and $30 \%$ glycerol. After vortex mixing for $3 \mathrm{~s}$ and degassing for $3 \mathrm{~min}, 1.2 \mu \mathrm{L}$ acetone were added, and photopolymerization was carried out for $28 \mathrm{~min}$ as described before (29).

To produce aldehyde groups, the gel was activated by incubation of the gel micromatrix in $0.1 \mathrm{M} \mathrm{NaIO}_{4}$ for $30 \mathrm{~min}$ at room temperature. The micromatrix was washed with water for $10 \mathrm{~min}$ and dried. The application and the immobilization of oligonucleotides were carried out as described before (29) for the standard gel.

\section{PCR Amplification Outside and Inside Microchip Gel Pads}

A microchip chamber for PCR amplification and hybridization was manufactured by placing a quartz cover glass $(20 \times 35 \times 3 \mathrm{~mm})$ over the microchip glass microscope slide. The 3.3 $\times 3.3-\mathrm{mm}$ microchip area of the slide is an array of $11 \times 11$ gel pads of $100 \times$ $100 \mu \mathrm{m}$ each. The quartz plate contains two 1-mm holes to fill the chamber with reaction mixtures through the attached polyethylene tubes. The glass and quartz plates were separated by an $18 \times 18$-mm plastic sticky spacer (In situ Frame; Eppendorf Scientific, Westbury, NY, USA), 63- $\mu \mathrm{m}$ thick, having a $9 \times 9$-mm hole for the microchip area. The sandwich was fixed by pressing two plates together and keeping for 4-5 min at $94^{\circ} \mathrm{C}$ according to the manufacturer's protocol. The chamber was filled with $30 \mu \mathrm{L}$ PCR buffer through a plate hole. The PCR buffer contained $2.5 \mathrm{mM} \mathrm{MgCl} 2,10 \mathrm{mM} \mathrm{KCl}, 10 \mathrm{mM}$ Tris- $\mathrm{HCl}, \mathrm{pH} 8.3,0.1 \%$ bovine serum albumin (BSA) (Sigma), $200 \mu \mathrm{M}$ each dNTP (Sigma), 5 U AmpliTaq ${ }^{\circledR}$ DNA polymerase, Stoffel Fragment of Taq DNA polymerase (Applied Biosystems, Foster City, CA, USA), 1-10 pmol each of the forward unlabeled and reverse labeled primers and $10^{4}$ copies of bacterial genomic or product DNA. Phage $\lambda$ DNA (100 ng; Sigma) was added to the PCR mixture when less than 10 ng DNA were used for amplification. After preheating at $95^{\circ} \mathrm{C}$ for 120 s, 25-35 cycles of PCR amplification were carried out at $95^{\circ} \mathrm{C}$ for $30 \mathrm{~s}$, at an- 


\section{Research Report}

Table 1. PCR Primers and Oligonucleotide Probes

\begin{tabular}{|c|c|c|c|}
\hline Oligonucleotidesa & Positions ${ }^{b}$ & Sequence $5^{\prime} \rightarrow 3^{\prime}$ & $\mathrm{T}_{\mathrm{an}}{ }^{\circ} \mathbf{C} \mathbf{c}$ \\
\hline lef- $\mathrm{F}$ & 1255 & СССТTGATAATATCTTACC & 51 \\
\hline lef- R & 1153 & GATATGAACCCGTACTTG & 51 \\
\hline pag4-F & 1931 & CAAGTTCCCAGGGGTTACTAGG & 58 \\
\hline pag4-R & 2178 & CACTTCTTGGTCATCTACCCAC & 58 \\
\hline pag4-P & 2155 & TTGTTACATGATTATCAGCGGAA & 58 \\
\hline bla-F & 2656 & CCGCCTCCATCCAGTCTATT & 58 \\
\hline bla- $\mathrm{R}$ & 2748 & CTGTAGCAATGGCAACAACG & 58 \\
\hline bla-F1 & 2682 & TGCCGGGAAGCTAGAGTAAGTAGTTC & 64 \\
\hline sht- $\mathrm{F}(\mathrm{T})$ & $1619 / 1648 \mathrm{~T}$ & TTCTTTGTTATCTTTTCAGTTAATGTGGTt & 67 \\
\hline$s / t-F(G)$ & $1619 / 1668 \mathrm{G}$ & TTCTTTGTTATCTTTTCAGTTAATGTGGTg & 67 \\
\hline sht- $\mathrm{F}$ & 1619 & TTCTTTGTTATCTTTTCAGTTAATGTGGT & 65 \\
\hline sht- $\mathrm{R}$ & 1757 & TACCTCCTGATGAAATAGTCTGTAATGG & 67 \\
\hline rpoB-F & 1275 & CGCGATCAAGGAGTTCTTCGGCACC & 68 \\
\hline$r p o B \mathrm{R}$ & 1377 & CCCGGCGGTCTGTACGTGA & 65 \\
\hline$r p o B I F 1 A$ & $1295 / 1323 \mathrm{~W}$ & GCACCAGCCAGCTGAGACAATTCATGGAC & 70 \\
\hline$r p o B I F 1 B$ & $1295 / 1322 A \rightarrow T$ & GCACCAGCCAGCTGAGACAATTCATGGt & 70 \\
\hline rpoBIF1C & $1295 / 1321 \mathrm{G} \rightarrow \mathrm{T}$ & GCACCAGCCAGCTGAGCCAATTCATGt & 70 \\
\hline$r p o B I F 1 D$ & $1295 / 1323 \mathrm{G} \rightarrow \mathrm{G}$ & GCACCAGCCAGCTGAGACAATTCATGGAg & 70 \\
\hline$r p o B I F 2 A$ & $1308 / 1336 \mathrm{~W}$ & GAGCCAATTCATGGACCAGAACAACCCGC & 70 \\
\hline$r p o B I F 2 B$ & $1306 / 1336 \mathrm{G} \rightarrow \mathrm{A}$ & CTGAGCCAATTCATGGACCAGAACAACCCGa & 70 \\
\hline$r p o B I F 3 A$ & $1310 / 1340 \mathrm{~W}$ & GCCAATTCATGGACCAGAACAACCCGCTGTC & 71 \\
\hline rpoBIF3B & $1310 / 1340 \mathrm{G} \rightarrow \mathrm{T}$ & GCCAATTCATGGACCAGAACAACCCGCTGTt & 71 \\
\hline rpoBIF 4A & $1323 / 1351 \mathrm{~W}$ & CCAGAACTACCCGCTGTCGTGGTTGACCC & 71 \\
\hline rpoBIF4B & $1323 / 1351 \mathrm{G} \rightarrow \mathrm{T}$ & CCAGAACTACCCGCTGTCGTGGTTGACCt & 71 \\
\hline rpoBIF4C & $1323 / 1351 \mathrm{G} \rightarrow \mathrm{G}$ & CCAGAACTACCCGCTGTCGTGGTTGACCg & 71 \\
\hline$r p o B I F 4 D$ & $1323 / 1351 \mathrm{C} \rightarrow \mathrm{A}$ & CCAGAACTACCCGCTGTCGTGGTTGACCa & 70 \\
\hline$r p o B I F 5 A$ & $1325 / 1352 \mathrm{~W}$ & AGAACTACCCGCTGTCGTGGTTGACCCA & 71 \\
\hline rpoBIF5B & $1325 / 1352 A \rightarrow G$ & AGAACTACCCGCTGTCGTGGTTGACCCg & 71 \\
\hline rpoBIF5C & $1325 / 1352 \mathrm{~A} \rightarrow \mathrm{T}$ & AGAACTACCCGCTGTCGTGGTTGACCCt & 71 \\
\hline rpoBIF5D & $1325 / 1352 \mathrm{~A} \rightarrow \mathrm{C}$ & AGAACTACCCGCTGTCGTGGTTGACCCc & 71 \\
\hline rpoBIF6A,7A & $1343 / 1367 \mathrm{~W}$ & GGTTGACCCACAAGCGCCGACTGTC & 70 \\
\hline rpoBIF6B,7B & $1343 / 1367 \mathrm{G} \rightarrow \mathrm{T}$ & GGTTGACCCACAAGCGCCGACTGTt(A+G) & 70 \\
\hline rpoBIF6C,7C & $1342 / 1367 \mathrm{G} \rightarrow \mathrm{G}$ & GGGTTGACCAACAAGCGCCGACTGTgt & 70 \\
\hline rpoBIF6D & $1342 / 1367 \mathrm{G} \rightarrow \mathrm{G}$ & GGGTTGACCAACAAGCGCCGACTGTgG & 70 \\
\hline rpoBIF7D & $1342 / 1367 \mathrm{G} \rightarrow \mathrm{A}$ & GGGTTGACCAACAAGCGCCGACTGTa(C+T) & 70 \\
\hline rpoBIF8A & 1349/1373 W & CCCACAAGCTCCGACTGTAGGCGCT & 71 \\
\hline$r p o B I F 8 B$ & $1349 / 1373 \mathrm{~T} \rightarrow \mathrm{C}$ & CCCACAAGCTCCGACTGTAGGCGCC & 71 \\
\hline \multicolumn{4}{|c|}{ a $F$, forward; $R$, reverse; IF, internal forward primers, respectively; $P$, hybridization probe. } \\
\hline \multicolumn{4}{|c|}{$\begin{array}{l}\text { b The first figure indicates the nucleotide position number, counting from the } 5^{\prime} \text { terminus of the gene-encoding sequence. } \\
\text { The second figure indicates the position of a mutated base. W, wild-type base. }\end{array}$} \\
\hline \multicolumn{4}{|c|}{$\begin{array}{l}{ }^{c} \text { Annealing temperature, } \mathrm{T}_{\mathrm{an}}=\mathrm{T}_{\mathrm{m}}-5 \ldots 6^{\circ} \mathrm{C} \text {. Melting temperature was calculated with Williamstone Software } \\
\text { (http://www.williamstone.com/) by the nearest-neighbor method. }\end{array}$} \\
\hline
\end{tabular}


nealing temperature for $60 \mathrm{~s}$ and at $72^{\circ} \mathrm{C}$ for $40 \mathrm{~s}$. The calculated annealing temperatures for the primers are shown in Table 1; however, the temperature used was $5^{\circ} \mathrm{C}-6^{\circ} \mathrm{C}$ lower because the gel decreases the melting temperature of duplexes (9). Thermocycling was carried out by placing the microchip chamber on the Peltier thermotable of a fluorescent microscope (9). The kinetics of amplification was monitored by measuring the fluorescent signal in parallel for all gel pads of the microchip.

The hybridization chamber was disassembled after amplification, and the microchip was washed with $300 \mu \mathrm{L}$ water at $95^{\circ} \mathrm{C}$ for $10 \mathrm{~min}$ and air-dried. The microchip-immobilized primers that had been extended during PCR amplification with $30 \mu \mathrm{L}$ Texas Red-labeled complementary probes $(1 \mathrm{pmol} /$ $\mu \mathrm{L})$ were hybridized in PCR buffer at $55^{\circ} \mathrm{C}$ for $0.5 \mathrm{~h}$. The melting curves of the duplexes formed were obtained with a fluorescent microscope (9) by increasing the temperature at the rate of $0.5^{\circ} \mathrm{C} / \mathrm{min}$.

\section{Fluorescence Measurements}

All experiments were performed in real time with an automatic experimental setup (9) consisting of a fluorescent microscope, a charge-coupled device (CCD) camera, Peltier thermotable, a temperature controller, a motion controller with step-motors and a computer equipped with a data acquisition board. The Special Hybridization Experiment Software package was designed for experimental control and data processing; the program uses Lab$\mathrm{View}^{\mathrm{TM}}$ software (National Instruments, Austin, TX, USA).

\section{RESULTS}

\section{Amplification in the Microchip Chamber}

The scheme of parallel amplification in solution and inside gel pads is shown in Figure 1. The DNA template solutions, forward and reverse primers (Figure 1A) and all other amplification components were placed above the oligonucleotide microchip in the hybridization chamber. The reverse primers were fluorescently labeled with Texas Red. The microchip contained the gelimmobilized forward primers, which were either the same as those in solution or complementary to an internal region of the amplified DNA (Figure 1, C-G).

The use of the second internal im mobilized primer allows one to carry out the second round of the gel amplification of the internal region of the DNA template amplified before in the solution. Such two-step reactions with different internal and external primers are similar to the nested primer amplification (23) and are used to increase the specificity of the procedure.

The large size of the DNA template limited its diffusion into the gel. Therefore, the initial rounds of amplification took place in solution (Figure 1A). The short amplified DNA fragment (Figure 1B), the labeled reverse primer, the DNA polymerase and all amplification components diffused into the gel pads of the microchip from the solution. The subsequent rounds of amplification occurred both in the solution (Figure 1B) and inside the gel pads and led to the extension of the immobilized forward terminal (Figure 1C) or internal (Figure $1 \mathrm{G})$ primers.

Amplification was monitored with the fluorescent microscope by measuring the hybridization signal of the extended immobilized primers with the fluorescently labeled amplified com plementary strand of the DNA fragment in each gel pad of the microchip (Figure 1, D and H). Alternatively, hybridization of the labeled internal probe with the extended forward primer can be used to monitor the amplification kinetics (Figure 1, A, B and G). The internal inactive probe was labeled through the $3^{\prime}$ end, which prevented its extension during the amplification procedure (Figure 1F).

To test the specificity of on-chip

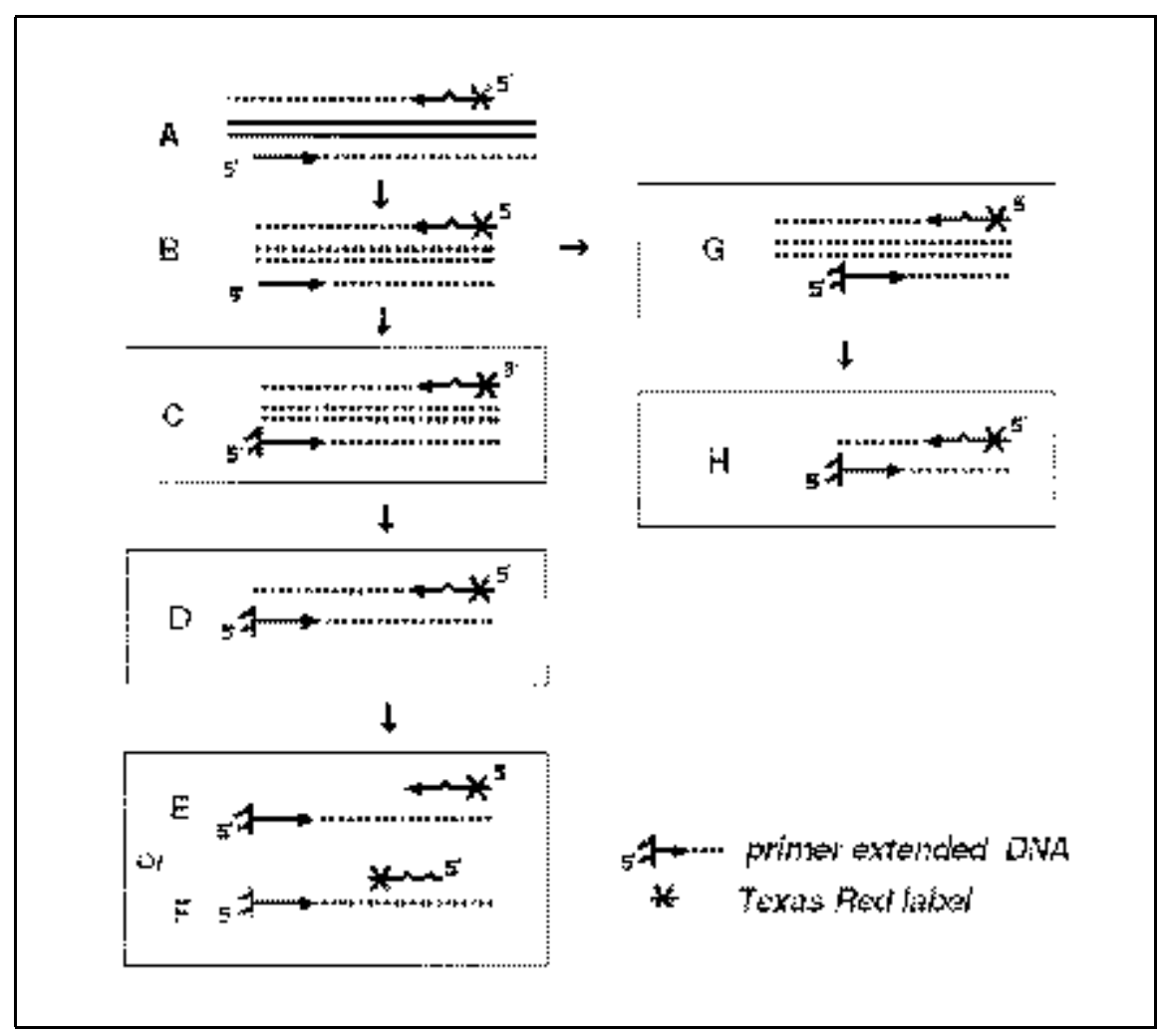

Figure 1. Scheme of PCR amplification over/on oligonucleotide microchips. The initial DNA was first amplified in solution over a microchip (A). The short fragment was further amplified over (B) and in the microchip gel pads on immobilized forward $(\mathrm{C})$ or internal $(\mathrm{G})$ primers. Labeled duplexes were formed by hybridization of the extended immobilized primers with the amplified fluorescently labeled complementary strand (D and $\mathrm{H}$ ) and were monitored in real time with the fluorescent microscope. The specificity of the reaction was tested by hybridization of the extended immobilized primers with the labeled reverse primer $(\mathrm{E})$ or internal probe $(\mathrm{F})$. Amplifications inside the gel pads on the immobilized primers are boxed. 
PCR amplification, the DNA duplexes were dissociated at high temperature, and the fluorescently labeled strands were washed off the gel pads. The remaining immobilized extended primers were then hybridized with the added fluorescently labeled reverse primers (Figure 1E) or with an internal oligonucleotide probe (Figure $1 \mathrm{~F}$ ) to provide an additional test for amplification specificity. The specificity of hybridization, and therefore of amplification, was controlled by measuring the melting curves of the duplexes formed in- side the gel pads.

Standard and porous (2) gels have been used for PCR amplification and are accessible to globular proteins of about $100 \mathrm{kDa}$ in size and to DNA of $100-150 \mathrm{bp}$ and about $400 \mathrm{bp}$, respectively, to diffuse into it. The standard gel pads sustained many cycles of amplifications at up to $95^{\circ} \mathrm{C}$ without any distortion and are easy to work with. Porous gels are more advantageous, providing the faster diffusion rate of large molecules and the higher level of amplification than the standard ones, but they are less stable and therefore need more careful treatment because some gel pads can be broken under the conditions of amplification. This caused about $10 \%$ failure of PCR amplification on porous gel microchips. More stable porous gels are under development in our group.

\section{Detection of the Anthrax Toxin Genes}

The PCR products containing fragments of the lethal factor (lef) and the
A

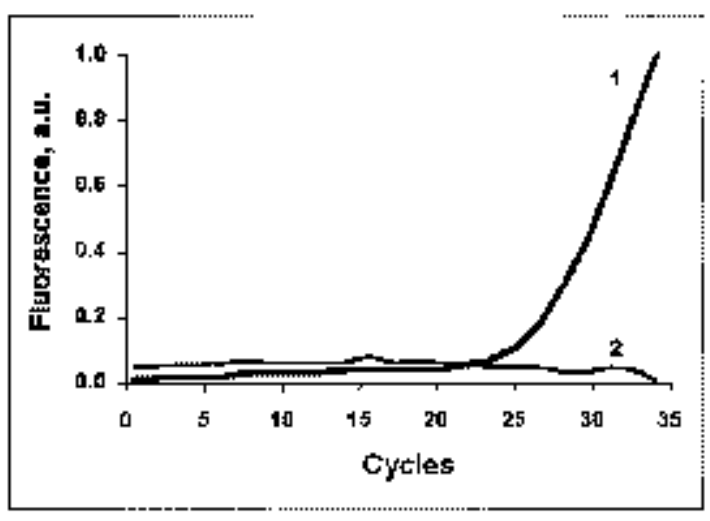

$\mathrm{C}$

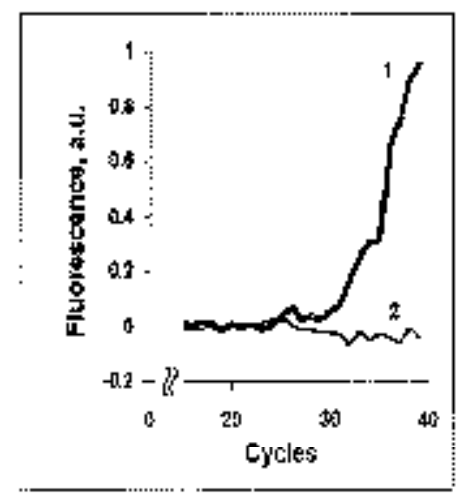

D

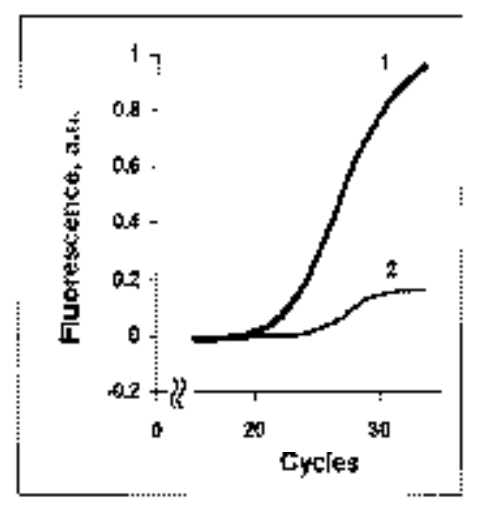

B

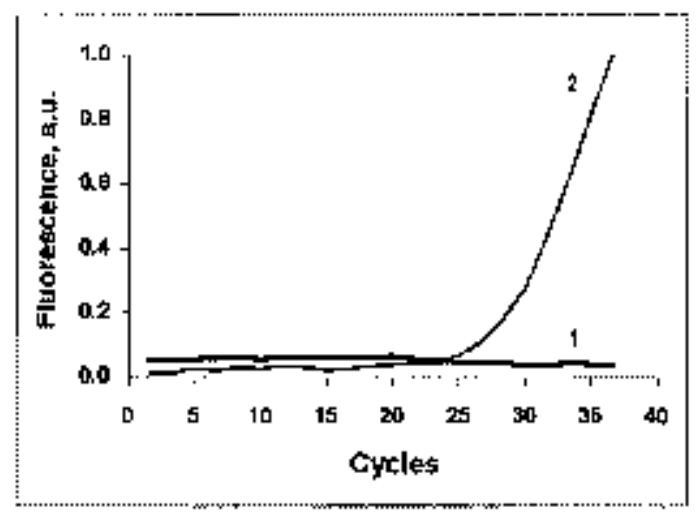

$\mathrm{E}$

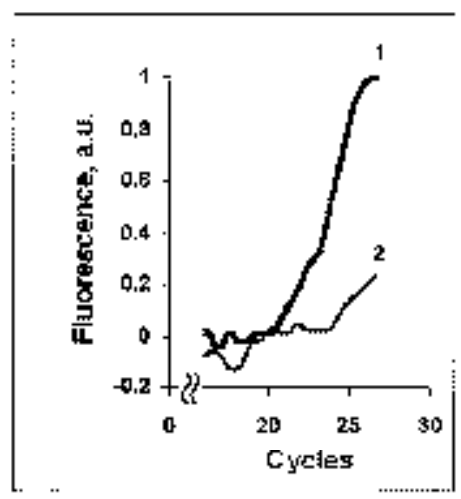

Figure 2. Monitoring PCR amplification of different DNA. (A and B) Amplification of the anthrax toxin gene fragments. Microchip gel pads contained the immobilized forward lef-F (see Table 1) or pag4-F primers. The PCR amplification solution contained the unbound forward lef-F and pag4-F primers, fluorescently labeled reverse lef-R and pag4-R primers and either lef or pag4 products. The kinetics of amplification was monitored in real time as the accumulation of fluorescently labeled duplexes formed by the extended immobilized lef-F primer (curve 1) or pag4-F primer (curve 2) and the complementary DNA strands of either lef (A) or pag4 (B) products. (C) Amplification of the pag4 gene fragment with internal labeled probe. The pag4 product was amplified as in Figure 2B, except that primers $l e f-\mathrm{R}$ and pag4-R were unlabeled. The $3^{\prime}$ Texas Red-labeled probe, pag4-P, for the internal product region, inactive as a primer, was added to the reaction mixture (see Figure 1F). The fluorescent signal increased in the gel pads containing pag4-F primers (curve 1) but not lef-F (curve 2). (D) Amplification of the ampicillin resistance bla-gene. The PCR amplification solution contained forward primer bla-F, labeled reverse primer bla-R and pUC18. Different gel pads of the microchip contained either forward terminal primer bla-F (curve 1), the same as in solution, or different internal primer bla-FI (curve 2). (E) Allele-specific PCR amplification of the Shiga toxin gene fragment with the immobilized sht-F(T) (curve 1) and slt-F(G) (curve 2) primers. For more details, see Figure 4 and the text. a.u., arbitrary units; one arbitrary unit is the maximal level of the fluorescent signal in each experiment. 
protective antigen (pag4) were used to detect the anthrax toxin genes. Two forward primers, lef-F and pag4-F, as well as two fluorescently labeled reverse primers, lef-R and pag4-R (see Table $1)$, were present in the amplification mixture containing either the lef or pag4 products. A standard polyacrylamide gel microchip contained lef-F and pag4-F immobilized in separate gel pads. Amplification led to the formation of duplexes between the immobilized extended primers and the amplified labeled complementary strand of the DNA fragments (Figure 1C). Figure $2, \mathrm{~A}$ and $\mathrm{B}$, shows the kinetics of am plification of the lef and pag4 fragments. The fluorescence of the gel pads was measured in real time and in parallel for all microchip elements. The exponential amplification of each DNA template was observed only for the gel pads containing the appropriate immobilized primers upon the 23rd and 25th cycle for the lef and pag4 genes, respectively. No significant fluorescence was detected for the pads containing inappropriate primers. The amplification coefficient for each cycle was about 1.89 , as determined by calculating the increase in the fluorescence intensity averaged over several cycles within the logarithmic phase of the amplification curve. The size of the amplified DNA was found to correspond to the expected length as verified by electrophoresis in a $2 \%$ agarose gel (not shown).

The specificity of on-chip amplification was evidenced by hybridization of the extended immobilized pag4-F primer with the corresponding internal probe (Figure 1F). The melting curve of the formed duplex is displayed in Figure 3 . The sharp decrease of the fluorescent signal within the narrow tem perature range points to the high cooperative interaction process, which indirectly confirms full complementarity of the probe and the amplified DNA.

Alternatively, the kinetics and specificity of amplification were tested by performing the reaction in the presence of unlabeled forward and reverse primers (Figure 1, A, B and C) and the 3 -labeled inactive probe for an internal region (Figure 1F) of the product. Figure $2 \mathrm{C}$ shows monitoring of the pag4 amplification by assessing hybridization of the fluorescently labeled inter- nal pag4-P probe with the extended im mobilized forward primer pag4-F. The exponential growth of fluorescence was observed upon the 26th round of amplification, which is two to three rounds later than in a similar experiment performed in the absence of the internal probe (Figure 2A).

\section{Detection of the $\beta$-Lactamase Gene}

The combined amplification outside and inside the standard polyacrylamide gel pads was also used to identify the $\beta$ lactamase (bla) gene in pUC18 responsible for bacterial resistance to ampicillin. Figure 2D presents the results of two amplification processes (Figure 1, $\mathrm{C}$ and $\mathrm{G}$ ) taking place in two gel pads of one microchip with the forward bla-F and reverse bla-R primers occurring in solution. However, one gel pad contained the immobilized bla-F, the same as in solution, while the other one contained the immobilized nested internal forward primer bla-FI to increase the specificity of amplification (Table 1).

However, the observed efficiency of the amplification was actually lower than that of the reaction carried out with the same forward primer present both in solution and within a gel. The cause of this effect is not clear. Among other factors, one could mention a nature of the primers or difference in lengths of the amplified fragments.

\section{Detection and Discrimination of the Shiga and Shiga-Like Toxin Genes}

The coding sequences of the Shiga $(s h t)$ and Shiga-like (slt) toxin genes differ in four point mutations. Therefore, detecting the Shiga toxin gene and discriminating it from the Shiga-like one should be performed in parallel with identifying the polymorphic nucleotides at least in one position. The T-to-G transversion at the 1648th nucleotide of the sht and slt genes was selected for the test. Modification of the allele-specific PCR amplification (20) was used to discriminate mutations. A scheme of the experiment is shown in Figure 4. The amplification solution contained the unlabeled forward primer sht-F (Table 1), the labeled reverse primer sht-R and pSHT23 (17). In sht-F, the $3^{\prime}$ terminal nucleotide is posi- 
tioned just before the polymorphic 1648th nucleotide (Figure 4A); therefore, amplification in solution with the use of these two primers should be sim ilar for both genes. Two forward primers, sht $\mathrm{F}(\mathrm{T})$ and slt-F(G), were immobilized in different pads of a porous gel and corresponded to the sht or slt genes, respectively. They overlapped the polymorphic nucleotide by one base (Figure 4B) and were derived from $s h t$-F by extension of its $3^{\prime}$ terminal nucleotide with either $\mathrm{T}$ or $\mathrm{G}$.

Figure 2E shows the kinetics of amplification of the sht gene on two shtand slt-immobilized primers. The amplification occurred much more efficiently on the fully complementary $3^{\prime}$ T-containing sht-immobilized primer sht-F(T) (Figure 4C). The kinetics of amplification was monitored by hybridization of the fluorescently labeled complementary DNA strand with the extended primer. The Stoffel fragment of Taq DNA polymerase was unable to extend efficiently the terminally mismatched slt immobilized primer slt-F(G), owing to the lack of exonuclease activity (Figure 4D). A fluorescent signal was still evident on the mismatched slt primer slt-F(G), which is probably explained by its terminally mismatched hybridization with the amplified labeled upper sht DNA strand. Thus, the difference in the kinetics of extension of the microchip-immobilized primers enables one to perform base polymorphism analysis in the course of gene identification.

\section{Identification of Rifampicin-Resis- tant M. tuberculosis Mutations}

All 39 identified rifampicin-resistant mutations were localized within a short 81-bp region of the rрoB gene encoding the $\beta$-subunit of RNA polymerase (19).

Multiplex allele-specific microchip PCR amplification $(4,28)$ with several immobilized primers was applied to identify some of these mutations. A scheme of these experiments (Figure 1, $\mathrm{A}, \mathrm{B}, \mathrm{G}$ and $\mathrm{H}$ ) was rather similar to those used to discriminate the Shiga and Shiga-like toxin genes (Figure 4, C and D). The genomic DNA of M. tuberculosis isolated from pulmonary sputum was first amplified outside a microchip in the hybridization chamber by using the forward primer rpoB-F (Table 1) and fluorescently labeled reverse primer rpoB-R. It produced a 123-bp fragment containing the region of the mutations (Figure 1A). The microchip, $3.3 \times 3.3$ $\mathrm{mm}$ in size, contained $11 \times 11$ gel pads and included 25 immobilized internal primers (Table 1). Among them, 16 primers corresponded to the mutations most frequently occurring in the Moscow region. The $3^{\prime}$ terminal nucleotides of these immobilized primers matched the variable mutated bases of the gene. The other six immobilized oligonucleotides corresponded to wildtype sequences and were used as internal controls for mutant base detection. The labeled complementary strand of the amplified DNA was hybridized with all multiplex primers of the microchip. However, only those primers that were complementary at their $3^{\prime}$ terminal position to the amplified DNA were efficiently extended by Stoffel DNA polymerase fragment (Figure 4C). The reaction with the terminally mismatched duplexes was at least five times less efficient (Figure 4D). The extended primers formed more stable duplexes during hybridization with the labeled complementary strand of the amplified DNA than the shorter unextended ones, and they could be easily discriminated at the increased temperature. This al-

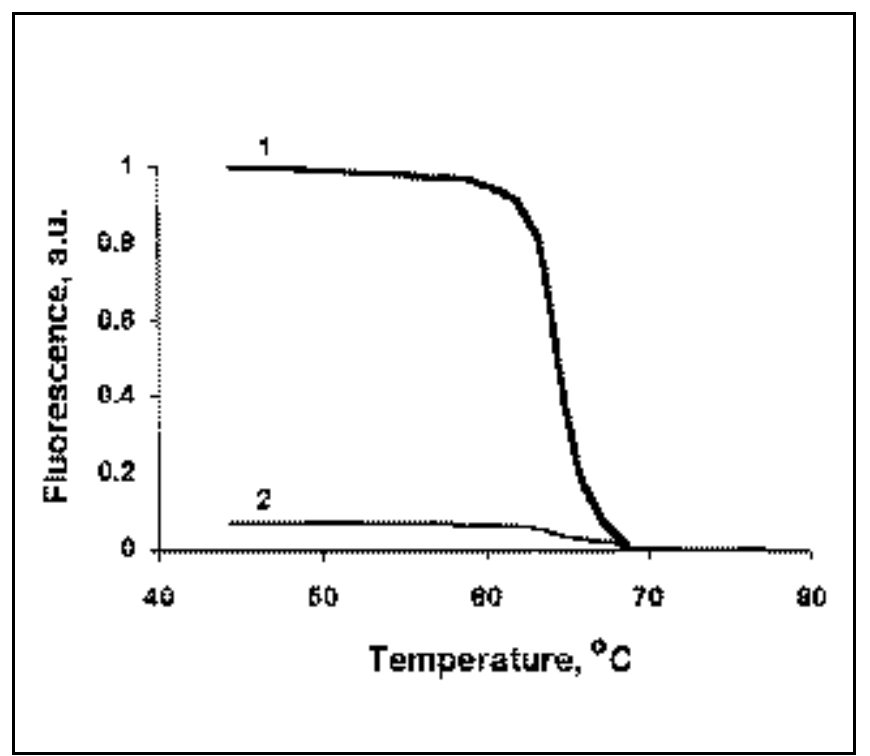

Figure 3. Melting curves of the duplex containing the extended pag4 microchip primer and pag4 internal labeled probe. After PCR amplification of the pag4 product (see Figure 2B), microchip duplexes were dissociated and lef-F (curve 2) and pag4-F (curve 1) extended immobilized primers were hybridized with the labeled oligonucleotide pag4-P probe specific to pag4 product (see Figure 1F). Melting curves were monitored in real time with the fluorescent microscope for both gel pads. Definition of arbitrary units is the same as in Figure 2.

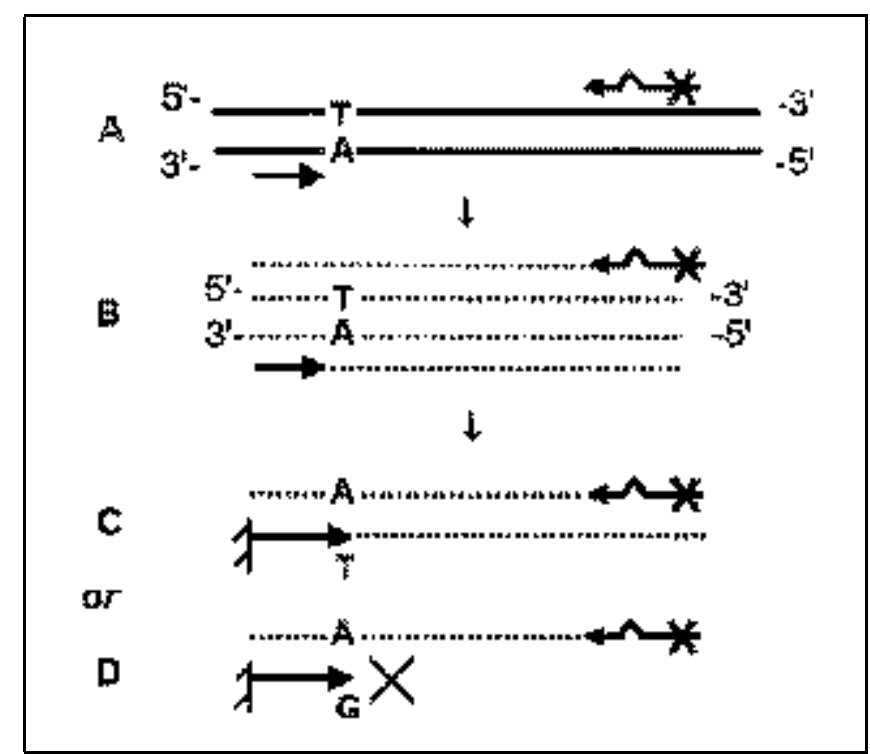

Figure 4. Scheme of allele-specific PCR amplification of the Shiga toxin (sht) gene fragment and discrimination of the T-containing Shiga from G-containing Shiga-like (slt) toxin gene. A specific region of the sht gene was amplified from the plasmid pSHT23 in solution with forward primer sht$\mathrm{F}$ and labeled reverse primer sht-R, which were complementary to both sht and slt genes. The microchip-immobilized two forward primers contained at the 3' terminal position either $\mathrm{T}$, sht $\mathrm{F}(\mathrm{T})$ or $\mathrm{G}$, slt $\mathrm{F}(\mathrm{G})$, corresponding to $\mathrm{T}$ or $\mathrm{G}$ at position 1648 of sht or slt genes, respectively. 


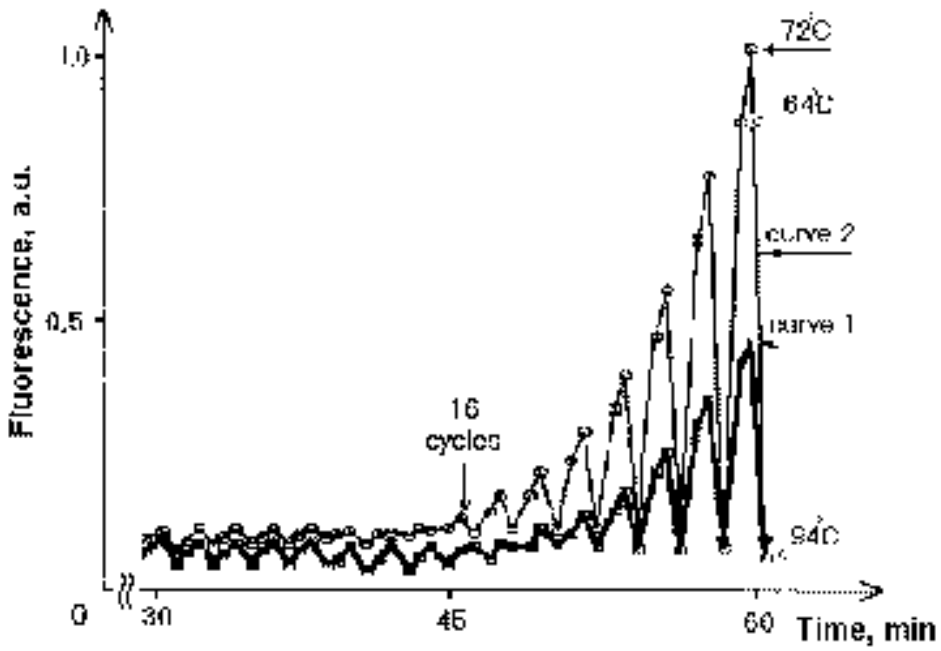

Figure 5. Monitoring the on-chip PCR amplification of the region of M. tuberculosis rpo Bgene containing rifampicin resistance mutations. The fluorescence measurements of the amplification of a mutated DNA from a clinical sample (sputum) were carried out in microchip gel pads 6A (curve 1) and 6B (curve 2), which contained the wild-type and CT mutation primers, respectively. Real-time kinetics measurements were carried out at the extension temperature of $72^{\circ} \mathrm{C}$ (upper points), annealing temperature of $64^{\circ} \mathrm{C}$ (middle points) and denaturation temperature of $-94^{\circ} \mathrm{C}$ (lower points) of PCR cycles. For details see Figure 6II and Table 1. Definition of arbitrary units is the same as in Figure 2.
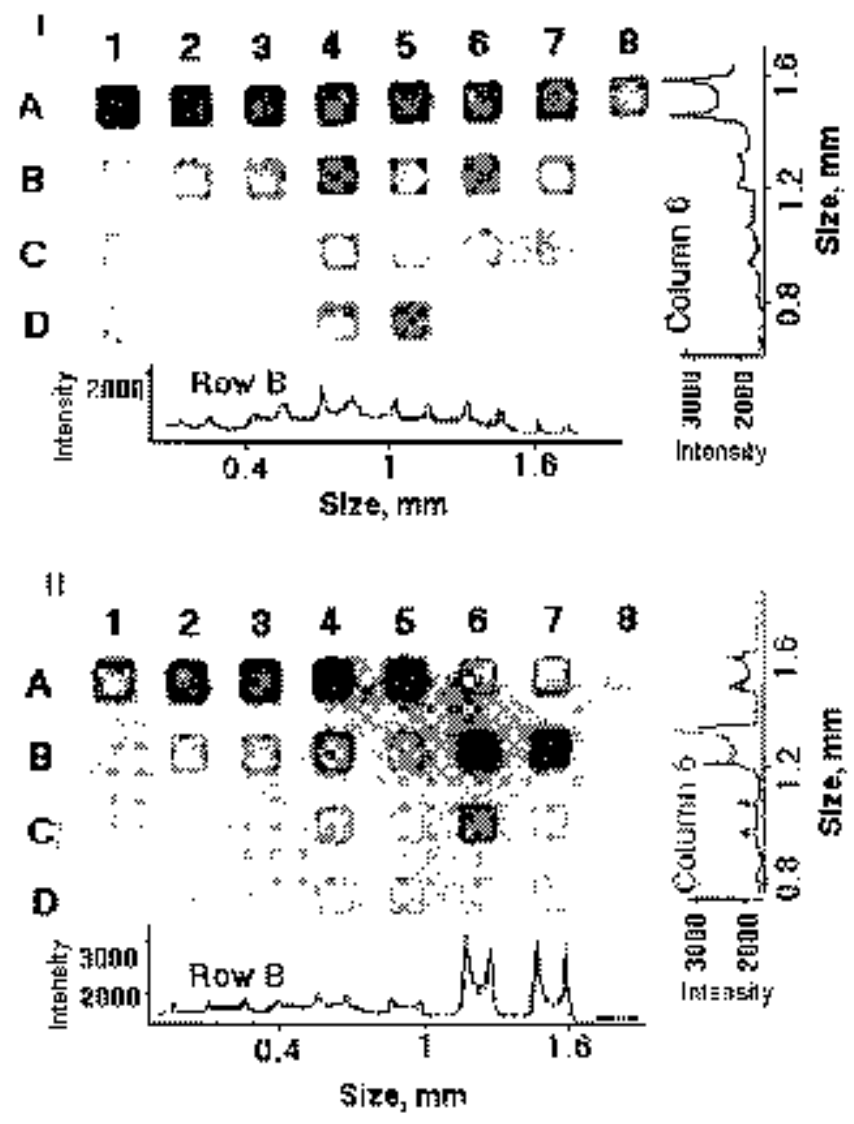

lowed us to monitor the reaction kinetics quantitatively in real time with the fluorescent microscope in the range of $72^{\circ} \mathrm{C}-85^{\circ} \mathrm{C}$ at the dissociation steps of amplification. Amplification kinetics of the mutated genomic DNA was measured for the gel pads containing the im mobilized primers for the mutated (rpoB-IF6B, Table 1) and wild-type (rpoB-IF6A) strains and is shown in Figure 5. Substantially weaker hybridization signals were observed for mismatched primers in pads with wildtype primers when mutated DNA was used as a template (curve 1 in Figure 5). This is probably explained by somewhat weaker hybridization of the amplified product with the unextended primers, as compared with the fully extended primer. During amplification, the duplexes formed by the unextended primers hybridized with the complementary labeled strand were dissociated in $0.3 \mathrm{M} \mathrm{NaCl}$ at $80^{\circ} \mathrm{C}$. However, the more stable duplexes formed by hybridization of the extended immobilized primers with fluorescently labeled com plementary strand remained inside gel pads and were quantitatively assessed. Figure 6 shows identification by this method of the $\mathrm{C} \rightarrow \mathrm{t} 1367$ mutation (see Table 1) causing the Ser 531 to Leu substitution in the RNA polymerase $\beta$-subunit. The ratio of the wild-type to mutant DNA in a sample might be measured by quantitative scanning of the corresponding gel pads (Figure 6).

Figure 6. Identification of rifampicin-resistant mutations in M. tuberculosis genomic DNA isolated from a clinical specimen (sputum) by allele-specific PCR amplification. The microchip contained the immobilized primers specific for the wild-type bacterial strain (the upper A row) and different mutations (lower B-D rows) arranged in separate columns (1-8). The sequences of these immobilized primers, indicated by the numbers and letters corresponding to their arrangement in the corresponding columns and rows, are shown in Table 1 . Note that the gel pads $6 \mathrm{~A}$ and $7 \mathrm{~A}$, as well as $6 \mathrm{~B}$ and 7B, contained identical immobilized primers. The mutations can be identified by comparing fluorescence intensities of the immobilized primers for wild-type (6A, $7 \mathrm{~A}$ ) and mutated sequences (6B and 7B) after the microchip amplification with wild-type (I) or mutant DNA (II). The microchip scans of row B and column 6 are shown below and on the right of the microchips, respectively. One axis indicates the fluorescence intensity as displayed by LabView program; the other axis is the size scale. 
The validity of this mutation identification was corroborated by direct sequencing. The procedure took about $1 \mathrm{~h}$ to perform and was sensitive enough to detect mutations in 100-1000 M. tuberculosis cells. Recently, this technique has been used successfully for the detection of 18 types of DNA mutations belonging to rifampicin-resistant $M$. $t u$ berculosis strains (B. Strizhkov, unpublished data).

Figure 7 compares PCR amplification inside the standard polyacrylamide and porous gel pads. The amplified fluorescently labeled duplexes fixed through immobilized extended primers were smoothly distributed within porous gels and located on the periphery of the standard gel pads. The porous gels also demonstrated the higher amplification signals.

\section{DISCUSSION}

The use of microchips containing gel-immobilized oligonucleotides for PCR amplification provides some essential advantages. The immobilization capacity of the gel pads exceeds that of solid surfaces such as glass by two orders of magnitude. Gel-immobilized compounds are in a more homogeneous water environment, which makes them more accessible for interaction with enzymes, as compared with solid-phase immobilized compounds. The amplification can be carried out simultaneously with the use of many primers immobilized in different gel pads. These factors, as well as the small size of the gel pads and a hybridization chamber, provide a higher sensitivity of measurements and a low consumption of reagents.

Immobilization in a gel of both forward and reverse primers for PCR am plification (bridge PCR) proposed by Mosaic Technologies (Waltham, MA, USA) (1) appears to restrict the flexibility of the DNA duplex fixed at both ends by these two primers. In addition, it significantly inhibited amplification (not shown; see also Reference 1). Therefore, in the microchip amplification experiments, only one primer was immobilized, while the other remained in solution.

The major part of the amplification took place in the solution above the microchip, as compared with the reaction within the gel pads. This accounts for the rather high (about 1.89) coefficient of the amplification for each cycle.

The over/on-chip amplification of

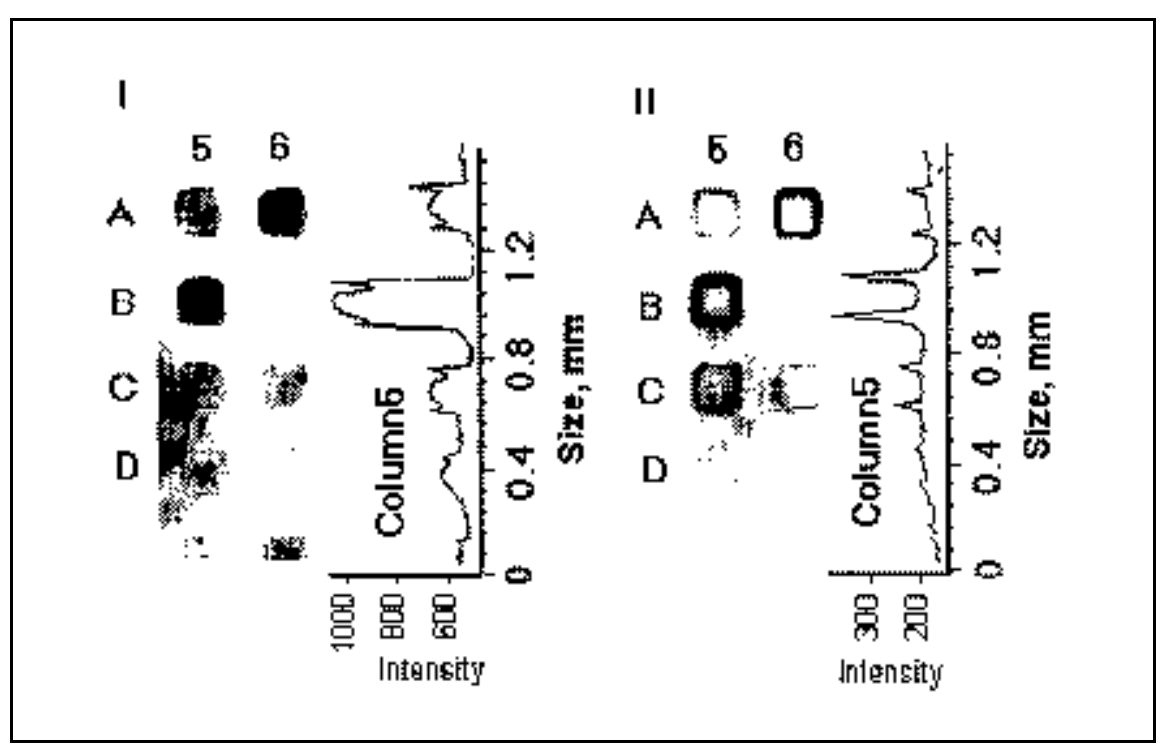

Figure 7. Comparison of PCR amplification on porous and standard gels with rpoBgene primers. The same microchip used in Figure 6II and another one prepared by immobilization of the same primers on the porous gel pads (I) were used in PCR amplification. DNA isolated from a patient contained the Ato-G substitution at the 1352 position of the rpoB gene, which corresponded to the His-to-Arg substitution in the protein. One can see a significant increase in the intensity of 5B pads for mutated DNA, as compared with the 5A pad hybridized with wild-type DNA (see Figure 6I). Designations of axes are the same as in Figure 6. longer DNA sequences was started in solution over the microchip and then proceeded in parallel with the shorter amplified fragments both in solution and inside the microchip gel pads. The forward primers and one reverse primer were in solution. Only one forward primer was immobilized in each gel pad for each amplified region. The am plification reagents, including the solution-amplified short DNA fragment, the reverse primer and DNA polymerase, rapidly diffused from solution into the tiny gel elements. Therefore, amplification occurred inside the gel pads as well. Diffusion of the reaction components into the gel slowed down the reaction for the standard gels to a higher extent than for the porous gels. Therefore, PCR amplification of the longer DNA fragments occurred mostly close to the surface of the standard gel but spread smoothly throughout the whole volume of porous gels. The microchip multiplex amplification was carried out with many primers for the $r p o B$ gene. Being immobilized in different gel pads, the primers did not interfere with each other (data not shown). For example, no cross-contamination of the oligonucleotides applied onto adjacent gel pads was observed in the course of microchip manufacturing (29).

Over/on-chip amplification and its allele-specific variation have integrated a number of analytical operations on one MAGIChip: $(i)$ the amplification proper; (ii) real-time monitoring reaction kinetics; (iii) highly sensitive and selective detection of the amplified products by using their hybridization with specific immobilized probes; and (iv) identification of genetic variants and mutations. These PCR and parallel hybridization procedures can be performed for $2 \mathrm{~h}$ with as few as 100-1000 DNA molecules. We have demonstrated application of over/on-biochip PCR amplification for identification of bacterial toxin genes as well as genes and genome mutations responsible for bacterial drug resistance. However, this approach shows promise for being easily extendable for many other important tasks.

The on-chip PCR amplification procedure is rather inexpensive because $(i)$ it requires very low amounts of reagents, (ii) the unit cost of biochips should be quite low upon mass produc- 
tion and (iii) a simple microchip analyzer (under \$2000 US) (A. Mirzabekov, unpublished data) can be used to analyze the hybridization pattern of fluorescently labeled amplified DNA with microchips containing tens of immobilized primers.

\section{ACKNOWLEDGMENTS}

We thank Drs. I. Taran and S. Surzhikov for oligonucleotide synthesis, E. Kreindlin and V. Chupeeva for microchip manufacturing, V. Barsky for expertise in the use of the fluorescent microscope and E. Timofeev and A. Kuhktin for advice in preparation of the standard polyacrylamide and porous gels. Dr. Yu. Kozlov kindly donated pSHT23. Thanks are also due to Drs. A. Moroz (Moscow Scientific Anti-Tuberculosis Center) for the $M$. tuberculosis clinical samples and John Ezzell (USAMRIID) for PCR-amplified fragments of the lef and pag4 genes. The authors are also indebted to Dr. S. Tillib for useful comments and to Dr. I. Roudykh and C. Kaicher for editorial assistance. This work was supported by the U.S. Department of Energy under contract no. W-31-109ENG-38, the U.S. Defense Advanced Research Project Agency under Interagency agreement no. AO-E428, by grant no. 5/2000 from the Russian $\mathrm{Hu}-$ man Genome Program and by grant no. a262 from the Moscow Government.

\section{REFERENCES}

1.Adams, C.P. and S.J. Kron. Bridge solid phase DNA amplification: method for performing amplification of nucleic acid with two primers bound to a single solid support. June 24, 1997, US Patent No. 5641658.

2.Arenkov, P., A. Kukhtin, A. Gemmell, V. Chupeeva, S. Voloschuk and A. Mirzabekov. 2000. Protein microchips: use for im munoassay and enzymatic reactions. Anal. Biochem. 278:123-131.

3.Belgrader, P., W. Benett, D. Hadley, G. Long, R. Mariella, Jr., F. Milanovich, S. Nasarabadi, W. Nelson, J. Richards and P. Stratton. 1998. Rapid pathogen detection using a microchip PCR array instrument. Clin. Chem. 44:2191-2194.

4.Dianzani, I., C. Camaschella, A. Pozone and R.G. Cotton. 1993. Dilemmas and progress in mutation detection. Trends Genet. 9:403-405.

5.Drobyshev, A., A. Zasedatelev, G. Yershov and A. Mirzabekov. 1999. Massive parallel analysis of DNA-Hoechst 33258 binding specificity with a generic oligodeoxyribonucleotide microchip. Nucleic Acids Res. 27:4100-4105.

6.Dubiley, S., E. Kirillov and A. Mirzabekov. 1999. Polymorphism analysis and gene detection by minisequencing on an array of gel-im mobilized primers. Nucleic Acids Res. 27:e19.

7.Dubiley, S., E. Kirillov, Y. Lysov and A. Mirzabekov. 1997. Fractionation, phosphorylation, and ligation on oligonucleotide microchips to enhance sequencing by hybridization. Nucleic Acids Res. 25:2259-2265.

8.Edwards, M.C. and R.A. Gibbs. 1994. Multiplex PCR: advantages, development, and applications. PCR Methods Appl. 3:S65-S75.

9.Fotin, A., A. Drobyshev, D. Proudnikov, A. Perov and A. Mirzabekov. 1998. Parallel thermodynamic analysis of duplexes on oligodeoxyribonucleotide microchips. Nucleic Acids Res. 26:1515-1521.

10.Gingeras, T., G. Ghandour, E. Wang, A. Berno, P. Small, P. Drobniewski, D. Alland, E. Desmond, M. Holodniy and J. Drenkow. 1998. Simultaneous genotyping and species identification using hybridization pattern recognition analysis of generic Mycobacteri$u m$ DNA arrays. Genome Res. 8:435-448.

11.Guschin, D.Y., B.K. Mobarry, D. Proudnikov, D.A. Stahl, B.E. Rittman and A.D. Mirzabekov. 1997. Oligonucleotide microchips as genosensors for determinative and environmental studies in microbiology. Appl. Environ. Microb. 63:2397-2402.

12.Heifets, L.B. 1996. The mycobacteriology laboratory. Past, present, and future. Clin. Lab. Med. 16:513-525.

13.Henderson, D.A. 1999. The looming threat of bioterrorism. Science 283:1279-1282.

14.Holland, P.M., R.D. Abramson, R. Watson and D.H. Gelfand. 1991. Detection of specific polymerase chain reaction product by utilizing the $5^{\prime} \rightarrow 3^{\prime}$ exonuclease activity of Thermus aquaticus DNA polymerase. Proc. Natl. Acad. Sci. USA 88:7276-7280.

15.Khalil, O.S. 1997. Automation and use of robotics in nucleic acid amplification assays. In H. Lee, S. Morse and O. Olsvik (Eds.), Nucleic Acids Amplification Technologies. Springer-Verlag, New York.

16.Kopp, M.U., A.J. de Mello and A. Manz. 1998. Chemical amplification: continuousflow PCR on a chip. Science 280:1046-1048.

17. Kozlov, Yu., A. Kabishev, E. Lukyanov and A.A. Bayev. 1988. The primary structure of the operons coding for Shigella dysenteriae toxin and temperature phage $\mathrm{H} 30$ shiga-like toxin. Gene 67:213-221.

18.Matsiota-Bernard, P., G. Vrioni and E. Marinis. 1998. Characterization of $r p o B$ mutations in rifampin-resistant clinical Mycobacterium tuberculosis isolates from Greece. J. Clin. Microbiol. 36:20-23.

19.Musser, J.M. 1995. Antimicrobial agent resistance in mycobacteria: molecular genetic insights. Clin. Microbiol. Rev. 8:496-514.

20.Newton, C.R., A. Graham, L.E. Heptinstall, S.J. Powell, C. Summers, N. Kalsheker, J.C. Smith and A.F. Markham. 1989. Analysis of any point mutation in DNA: the amplification refractory mutation system (ARMS). Nucleic
Acids Res. 17:2503-2515.

21.Proudnikov, D., E. Kirillov, K. Chumakov, J. Donlon, G. Rezapkin and A. Mirzabekov. 2000. Analysis of mutations in oral poliovirus vaccine by hybridization with generic oligonucleotide microchips. Biologicals 28:57-66.

22.Proudnikov, D., E. Timofeev and A. Mirzabekov. 1998. Immobilization of DNA in polyacrylamide gel for the manufacture of DNA and DNA-oligonucleotide microchips. Anal. Biochem. 259:34-41.

23. Rabodonirina, M., L. Cotte, A. Boibieux, K. Kaiser, M. Mayencon, D. Raffenot, C. Trepo, D. Peyramond and S. Picot. 1999. Detection of Pneumocystis carinii DNA in blood specimens from human immunodeficiency virus-infected patients by nested PCR. J. Clin. Microbiol. 37:127-131.

24.Shi, M.M., M.R. Bleavins and F.A. De La Iglesia. 1999. Technologies for detecting genetic polymorphisms in pharmacogenomics. Mol. Diagn. 4:343-351.

25.Taylor, T.B., E.S. Winn-Deen, E. Picozza, T.M. Woudenberg and M. Albin. 1997. Optimization of the performance of polymerase chain reaction in silicon-based microstructures. Nucleic Acids Res. 25:3164-3168.

26.Tyagi, S. and F.R. Kramer. 1996. Molecular beacons-probes that fluoresce upon hybridization. Nat. Biotechnol. 14:303-308.

27. Wilding, P., L.J. Kricka, J. Cheng, G. Hvichia, M.A. Shoffner and P. Fortina. 1998. Integrated cell isolation and polymerase chain reaction analysis using silicon microfilter chambers. Anal. Biochem. 257:95-100.

28.Wu, D.Y., L. Ugozzoli, B.K. Pal and R.B. Wallace. 1989. Allele-specific enzymatic am plification of $\beta$-globin genomic DNA for diagnosis of sickle cell anemia. Proc. Natl. Acad. Sci. USA 86:2757-2760.

29. Yershov, G., V. Barsky, A. Belgovskiy, E. Kirillov, E. Kreindlin, I. Ivanov, S. Parinov, D. Guschin, A. Drobyshev, S. Dubiley and A. Mirzabekov. 1996. DNA analysis and diagnostics on oligonucleotide microchips. Proc. Natl. Acad. Sci. USA93:4913-4918.

Received 24 March 2000; accepted 20 June 2000.

\section{Address correspondence to: \\ Dr. Andrei D. Mirzabekov \\ Biochip Technology Center \\ Argonne National Laboratory \\ 9700 South Cass Avenue \\ Argonne, IL 60439, USA \\ e-mail:amir@everest.bim.anl.gov}

